\title{
Xenon-Water Interaction in Bacterial Suspensions as Studied by NMR
}

\author{
Victor Rodin ${ }^{1, *}$, Alexander Ponomarev², Maxim Gerasimov ${ }^{3}$, Leonid Gurevich ${ }^{4}$ \\ ${ }^{1}$ Institute of Organic Chemistry, Johannes Kepler University Linz, Altenbergerstraße 69, 4040 Linz, Austria \\ ${ }^{2}$ Department of Medical Cell Technologies, Ural State Medical University, Ekaterinburg, Russian Federation \\ ${ }^{3}$ Department of Clinical and Experimental Medicine, Linköping University, Linköping, Sweden \\ ${ }^{4}$ Department of Physics and Nanotechnology, Aalborg University, Aalborg, Denmark \\ *Corresponding Author: victor.rodin@jku.at
}

Copyright $\subset 2017$ by authors, all rights reserved. Authors agree that this article remains permanently open access under the terms of the Creative Commons Attribution License 4.0 International License

\begin{abstract}
Xenon is a perspective gas for creation of oxygen free environment for different applications of biomaterials. To use xenon in suspensions and products properly it is necessary to know the molecular mechanisms of its interactions with water and cells. This work reports the study of bacterial suspensions of Escherichia coli in the presence of xenon using nuclear magnetic resonance (NMR). The work studied how the spin-lattice relaxation times of water protons in suspension change under xenon conditions. Xenon is able to form clathrate hydrates with water molecules at a temperature above the melting point of ice. The work studied NMR relaxation times which reflect the rotation freedom of water molecules in suspension. Lower relaxation times indicate reduced rotational freedom of water. Single exponential behavior of spin-lattice relaxation of protons in the suspensions of microorganisms has been registered. A recovery of longitudinal magnetization in cell suspensions with xenon clathrates has been characterized by two peaks in $T_{1}$-distribution. Fast relaxing $T_{1}$-component was related to the intracellular water and depended on the amount of xenon clathrates. The obtained results elucidate how the NMR method can monitor the process of clathrate formation and how the xenon atoms and hydrates interact with cells.
\end{abstract}

Keywords Bacterial Suspensions, Water, Xenon, Clathrates, Nuclear Magnetic Resonance

\section{Introduction}

Despite their inertness, noble gases possess a number of interesting properties, including their anaesthetic effect [1-4]. They are very soluble in lipids and may bind to both membrane lipids and proteins [3-5]. Because of the high correlation between lipid solubility and anaesthetic potency (known as the Meyer-Overton correlation) it was suggested that the molecular mechanisms of general anaesthetics are associated with the interactions between anaesthetic agents and a hydrophobic interior of the lipid bilayers [2, 4, 5]. Although different theories have been discussed in the literature for long time, the exact mechanism of anaesthetic action of noble gases is still a mystery $[5,6]$. It is not clear which of the different mechanisms suggested is most likely to be the main mechanism of action of anaesthetic agents.

Xenon is the most potent anaesthetic of all the noble gases. The high polarizability of xenon compared to the other noble gases increases its affinity to hydrophobic environments. Thus xenon can be substantially partitioned into the hydrophobic core of the membrane bilayer. The solubility of xenon in human serum albumin solutions, hemoglobin solutions, human blood, and other biological solutions and homogenates was studied in the Ref. [7]. For example, at 298 $\mathrm{K}$ the amount of xenon dissolved in $1 \mathrm{~g}$ human albumin at $0.1013 \mathrm{MPa}$ of total pressure was found to be $0.2382 \mathrm{ml}$. According to the data [3] a blood-gas partition coefficient for xenon is 0.115 whereas the solubility in water measured identically was 0.096 .

The anaesthetic gas xenon is attractive for many applications in biotechnology and medicine [8-10]. Xenon is also the perspective gas for preservation of biological materials including different enzymes and cells [11-16]. This noble gas is able to replace lighter gases of air, e.g. oxygen and nitrogen $[17,18]$. This inert gas was already applied for many biological and medical systems in creation of oxygen free environment, in particular, for bacterial cell suspensions and erythrocytes of blood [9, 10, 19-24]. The authors of Refs. [19, 20] developed the method of platelet storage in the presence of xenon. In order to use the xenon in the storage of biological suspensions and biotechnological products properly it is necessary to know the molecular mechanisms of its interactions with cell membrane and cell components $[5,11,13,21]$. Nowadays many questions are still opened. 
One important feature of xenon is its ability to create clathrate hydrates from water molecules at relatively "soft" conditions when the pressure is still about $0.4-0.7 \mathrm{MPa}$ and temperature circa 276-278 K [12-14, 21]. When forming hydrates, lower hydrate formation pressures are desirable in practice $[21,25,26]$. It is known that crystalline hydrates are formed by many simple gases that do not interact strongly with water, and mostly the gas molecules or atoms occupy "cages" formed by the network of water molecules. The majority of these gas hydrates adopt one of two possible cubic cage structures and therefore are called clathrate hydrates $[21,25,26]$. Although high pressure is often needed to hold gas atoms inside the water "cages", xenon gas is an exception from the rule. Xenon hydrates are the clathrates of low pressure. They can be stabilized at about 276-280 K [10, 12, 25].

Clathrate hypothesis of anaesthesia of L.Pauling [27, 28] suggested the existence of clathrate microcrystals formed by anaesthetic gas and water molecules even at physiological conditions (at least momentarily): normal atmospheric pressure and temperature of cells. Some suggestions of clathrate theory of L.Pauling have been checked later in Refs. [27-33]. First studies on formation of xenon clathrates in cellular suspensions of microorganisms have been initiated in Refs. [9, 13, 14, 34, 35]. Those publications showed how the methods of magnetic resonance can produce valuable information about the changes in bacterial medium under gas pressure. Later publications [21, 36-40] reported more NMR results on xenon clathrates. The use of paramagnetic additives expanded the possibilities of NMR relaxation in studying cell suspensions with gas hydrates [21, 36]. NMR was also applied for studying clathrate formation in twocomponent and complex systems [9, 37-40]. Nevertheless many questions about the role of xenon and its clathrates in cell suspensions still remain open.

The most intensively investigated prokaryotic model organism in biophysics is the Escherichia coli (E.coli), K-12 strain [41, 42]. It is considered as very important representative of cellular suspensions in biotechnology. E.coli is a common gram-negative rod-shaped bacterium, found in normal human (and other animals) bacterial flora. It is about $2.0 \mu \mathrm{m}$ in length and its diameter is $0.25-1.0 \mu \mathrm{m}$ [41]. These microorganisms are facultative anaerobic bacteria which can be easily grown in a laboratory.

The current work reports NMR-study of bacterial suspensions of E.coli under xenon pressure addressing how the xenon and its hydrates participate in the interactions with cells and affect the state of intra- and intercellular water. The biomaterials with crystals of xenon clathrates are "transparent" for radio waves. This allows using the methods of radio spectroscopy (i.e., NMR) to analyze the structural and phase state and molecular-dynamic properties of water in the cell suspensions in the presence of a clathrate phase. The paper presents how NMR water proton spin-lattice relaxation times in E.coli suspensions vary under xenon conditions.

\section{Experimental Materials and Methods}

\subsection{Materials}

The cells of E.coli have been grown in flasks with volume of 0.75 liter using beef-extract broth or mineral liquid medium with glucose. A sterilized phosphate buffered saline (PBS) was added. The cells at the stationary phase of growth were used (age of 14-16 hrs). The samples with cells were centrifuged at $3000 \mathrm{rpm}$ for $15 \mathrm{~min}$. The precipitate was washed twice in distilled water, PBS and was used in subsequent experiments as in Refs. [9, 21, 36]. A concentration of the biomaterial (cellular suspensions) under study was expressed in $\mathrm{g}$ of dry mass per $\mathrm{g}_{2} \mathrm{O}$ and by a number of cells per $1 \mathrm{ml}$ [21].

\subsection{Custom-made Thermostat Chamber with Xenon Kit}

We designed and built thermostat chamber with transparent front door from polymethyl methacrylate. Inside the chamber a system to transfer the gas to tubes with samples was built. The gas cylinder (with medical grade xenon) was connected to the special kit with tubes filled by suspensions / biomaterials [21, 27, 36]. The tubes with samples were under gas pressure as described in earlier publications [21, 37]. Mostly the pressure circa $0.8-1.0 \mathrm{MPa}$ or less was enough to produce stable clathrates at $278-280 \mathrm{~K}$. In some experiments "dry" ice (carbon dioxide) was used to touch the bottom of the tubes for the initiation of clathrate formation according to described procedure [21, 36, 43]. After saturation of the samples with gas and xenon clathrate formation the tubes could be detached from the kit in the thermostat chamber and be used in NMR measurements at above melting point of common ice but not exceeded $286 \mathrm{~K}$.

\subsection{NMR Methods in Studying Water Clathrates of Xenon}

The NMR studies of cell suspensions and suspensions with clathrate crystals were performed at a proton resonance frequency of $90 \mathrm{MHz}$. Some experiments have been carried out also at $20 \mathrm{MHz}$. In the single-pulse ${ }^{1} \mathrm{H}$ NMR experiments, the free induction decay (FID) signals were measured. The FID consists of a $90^{\circ}$ pulse which rotates the magnetization onto the transverse plane where it decays with characteristic time. The initial part of the FID is hidden in the dead-time zone of the spectrometer. The length of the $90^{\circ}$ pulse was 8 $\mu \mathrm{s}$. The FIDs on the samples with clathrates have been measured according to the procedure of earlier publications $[27,37,43]$. The signal of total time-domain is the sum of the signals of the water protons and the protons of clathrate crystals: $S=S_{\text {wat }}+S_{\text {clath. }}$. In the study of water dispersions of xenon clathrates [21,37] it was shown that spin-spin relaxation time $T_{2}$ of the fast relaxing component (protons of clathrates) did not exceed $25 \mu \mathrm{s}$. The structure of clathrate ice in studied solutions and suspensions at above melting point of common hexagonal ice was relatively "softer" than that in common ice with $T_{2} \sim 10 \mu \mathrm{s}$. For ice protons the most 
part of FID was hidden in the dead-time zone. Only the tail of the transverse magnetization decay might be observable in the experiment. Although the part of FID for the clathrates is a bit longer than the FID for common ice, the situation with FID in clathrates is comparable with that in ice. To monitor the water signal only, the contribution of the protons of clathrate ice should be subtracted. In such a case, normally, the measurements were carried out at experimental conditions when transverse magnetization of clathrate protons decayed very fast to zero (whereas the transverse magnetization of liquid water has not decayed) and only protons of water could be measured in the study $[37,43]$. On the base of this approach a fraction of water built in clathrate structures was determined using the difference of the proton signals of liquid water in suspensions before and after clathrate formation [21].

The spin-lattice relaxation time $T_{1}$ was measured by the inversion recovery (IR) method using the pulse sequence $\left(180^{\circ}-t-90^{\circ}\right)$ at the pulse sequence repetition time $\geq 5 T_{1}[45$, 46]. The period of $5 T_{1}$ was waited between the consecutive scans of pulse sequence in order to ensure that the fully recovered signal was acquired. When $t$ is varied then experimental dependence of magnetization $M_{\mathrm{t}}=f(t)$ can be obtained and fitted by the law $M_{\mathrm{t}}=\sum M_{0 \mathrm{i}} \cdot\left[1-2 \cdot \exp \left(-t / T_{1 \mathrm{i}}\right)\right]$, where $i$ is the number of relaxation component. The analysis of relaxation data was conducted after transferring the raw data from spectrometer computer to desk computer using in-house $M a t L a b \circledast$ codes. In these NMR studies there was an aim to model experimental data as a sum of several relaxation components or distribution of NMR-relaxation times. To fit the data inverse Laplace transform (ILT) in one direction was commonly applied [47-50]. The kernel function in ILT was associated with $M_{\mathrm{t}}$ data obtained from IR NMR experiments.

\section{Results and Discussion}

\subsection{Spin-lattice Relaxation of Water Protons in Suspensions of E.coli before and after Xenon Clathrate Formation}

The $T_{1}$-experiments discovered that the recovery of longitudinal magnetization of measurable protons in all studied samples of cell suspensions (concentration range of 0.001-0.24 g dry mass $/ \mathrm{g} \mathrm{H}_{2} \mathrm{O}$ ) can be fitted by single exponential curve and characterized by a single value of the spin-lattice relaxation time $T_{1}$. However a recovery of longitudinal magnetization of protons in bacterial suspensions with clathrates of xenon has bi-exponential behavior. $T_{1}$ values of the two exponential components differ by a factor of 6 to 10 . Alternatively, the treatment of the recovery of longitudinal magnetization using inverse Laplace transform resulted in a distribution of spin-lattice relaxation times $T_{1}$ in the kind of 2 peaks. Fig. 1 shows $T_{1}$-distributions obtained by one- dimensional ILT applied to the data sets for original bacterial suspension and E.coli with xenon clathrates.

The two peaks registered for the suspension of E.coli with xenon clathrates (Fig.1) had maximums at 0.25 and $1.2 \mathrm{~s}$. The integral intensities of these peaks corresponded to $66 \%$ and $34 \%$ of measurable protons. The single peak observed in $T_{1}$-distribution in suspension of E.coli without clathrates was at $T_{1} \approx 0.70 \mathrm{~s}$ (Fig.1). This was in line with previously published NMR relaxation data on cellular suspensions [21, 40] which showed fast exchange between the protons of intra- and intercellular water, i.e., single exponential $T_{1}$ -relaxation [51-53]. The permeability of the cell membrane to water is quite high (for instance, in red blood cells exchange time of the order of $10 \mathrm{~ms}$ [53]). Water proton relaxation times in E.coli bacteria showed one exponential behavior, i.e., two sites (two compartments: intracellular and extracellular spaces) are in a fast exchange limit. Thus a researcher needs to reduce one of the relaxation times to less than exchange time in order to remove this condition and to generate two exponential behavior [51, 52]. In the presence of paramagnetic ions of manganese in the extracellular water shortening the relaxation times outside the cells it was possible to record two separate NMR signals in the population of cells with non-damaged cellular membranes $[36,51,52]$.

The relaxation rates of the water molecules in cell suspensions are governed by several important factors, in particular, the strength of local magnetic interactions between water nuclei, the molecular motion and proton exchange rates. Nuclear magnetic inter- and intramolecular dipole-dipole coupling describes the interactions between water nuclei [51-53]. The magnetic interactions are partially averaged within the hydration layer by the processes depending on the interactions of water with macromolecules/cell surface/clathrates. These are proton transfer, dynamic orientation and diffusion of water molecules through regions of different orientations [26, 40, $44,53]$. The NMR relaxation times are sensitive to molecular motions in the range of $10^{-8}$ to $10^{-12} \mathrm{~s}[45,46,53]$. In liquid solutions water molecules tumble at a rate of about $10^{-12} \mathrm{~s}$ [45]. This motion is considerably slowed down when water molecules interact with biological macromolecules/cell surface. For example, the correlation time is of the order of $10^{-12} \mathrm{~s}$ for the water molecules non-involved in the association through hydrogen bonds with surface of cells. The rotational motion of water molecules associated via hydrogen bonds with polar groups of the macromolecules is reduced so that their correlation time is even sometimes of the order of $10^{-6} \mathrm{~s}$ [53]. Under the conditions of rapid exchange between the hydration and bulk water in suspensions and macromolecular solutions, single relaxation rate $\left(1 / T_{1}\right)$ is observed.

Single exponential relaxation of protons in suspensions of E.coli confirmed fast exchange between intracellular and intercellular water on the NMR time scale. Formation of xenon clathrates affected the exchange rate and resulted in the measurement of two separate compartments. The population of fast relaxing $T_{1}$-component was associated with the fraction of intracellular water. It depended on the amount of xenon clathrates.

Fig. 2 shows how the amount of clathrate ice, i.e. the amount of water molecules bounded within the structure of 
xenon clathrates, depends on the concentration of cells in suspension. The fraction of clathrates has been calculated according to the procedure [37-40] which compares the measurements of the FID in original bacterial suspension and suspension with xenon clathrates. The transition of liquid water into solid phase of clathrates resulted in a change of spin-spin relaxation times $T_{2}$ of protons. $T_{2} \sim 1-2 \mathrm{~s}$ for free mobile water whereas $T_{2} \sim 10 \mu$ s for protons of solid phase. Such a difference in $T_{2}$-values gives an opportunity to separate proton signals of liquid and solid phases much better. The results of Fig. 2 are in line with the previously published data on the xenon clathrates formation in solutions of biomacromolecules [12, 27, 31, 32, 34]. It was shown [31, $32,34]$ that a water fraction bound in clathrate structures was due to abundance of hydrophobic groups on the surface of proteins. Probably some groups on the cellular surfaces also participate in the formation of xenon hydrates stabilizing clathrate lattice from water molecules [21,28,31]. As a result the amount of stable clathrate structures was increasing with cell concentration in studied range. One observed that the amplitude of fast relaxing $T_{1}-$ component (relatively to total water content in cell suspension) increased nonlinearly with an increase in the initial cell concentration. Apparently this is due to the advent of solid phase of water (clathrates) and subsequent displacement of the microorganisms in the free volume of the suspension.

Fig.3 shows calculated fraction of intracellular water (open circles) and the dependence of the population of the fast relaxing $T_{1}$-component on cell concentration recalculated to the signal of liquid water. This recalculation shows the true concentration of the cells remaining in the liquid phase of the suspension after the formation of clathrates. The dependence of the population of this $T_{1}$-component looks almost linear, and this assumes that the fast component of $T_{1}$ is due to the protons of intracellular water. The slow $T_{1}$-component can be attributed then to the extracellular water protons. This assignment is further supported by a decrease of the population of the slow component with increasing concentration of cells. A fraction of the extracellular water protons which are not in clathrate structures decreased. According to this analysis, the cell concentration increased relatively to volume of liquid phase after clathrate formation. A fraction of intracellular water (Fig. 3, open circles) was calculated on the base of summary volume of cells and had a linear dependence on concentration of cells.

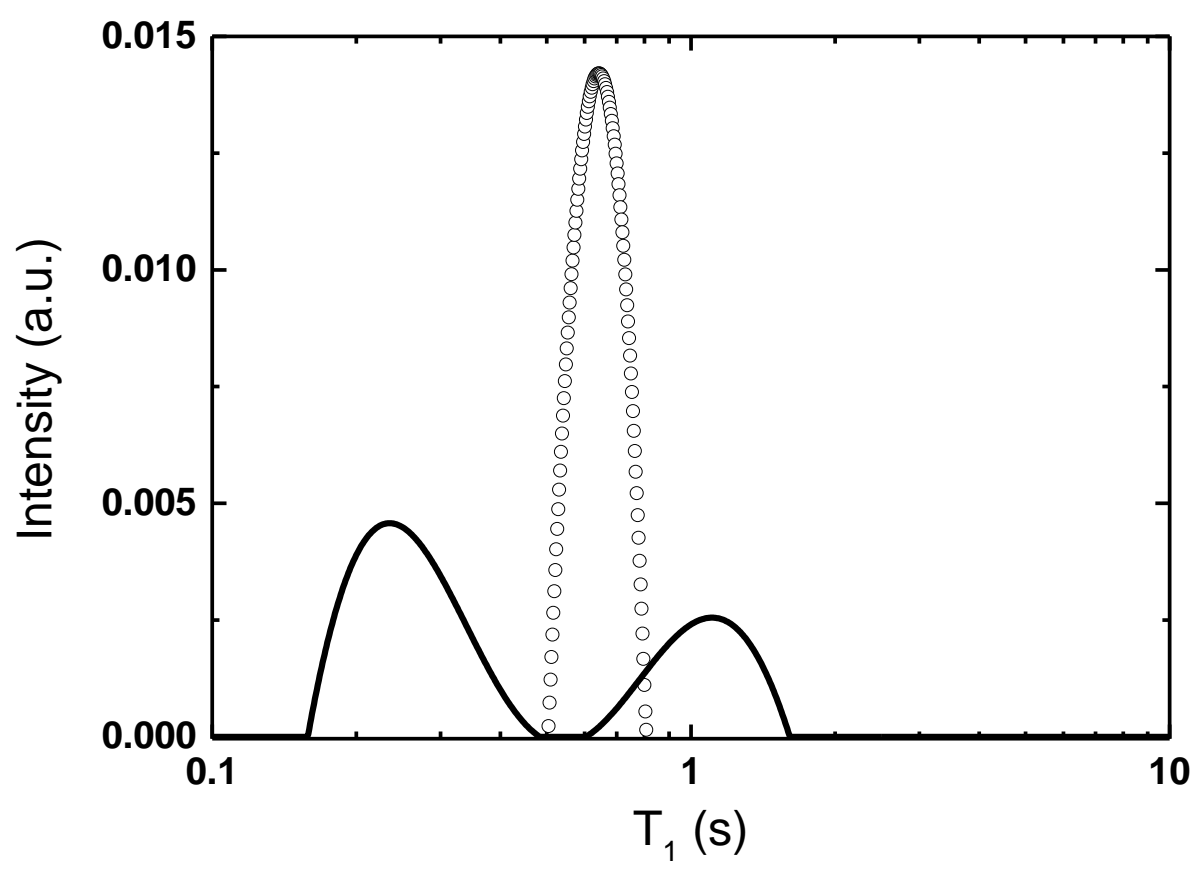

Figure 1. $T_{1}$-distribution of ${ }^{1} \mathrm{H}$ obtained by one-dimensional ILT on the recovery of longitudinal magnetization data set measured in the suspension of E.coli with concentration of $0.07 \mathrm{~g}$ dry mass $/ \mathrm{g} \mathrm{H}_{2} \mathrm{O}$ (open circles) and in the same sample after xenon clathrate formation at $1.2 \mathrm{MPa}$ (solid line). $\mathrm{T}=280 \mathrm{~K}$. Frequency is $90 \mathrm{MHz}$.

The integral intensities of 2 peaks in the suspension of E.coli with xenon clathrates (from left to right) are $66 \%$ and $34 \%$ of measurable protons. The signals in each $T_{1}$-experiment have been normalized per maximal signal registered after total recovery of longitudinal magnetization. 


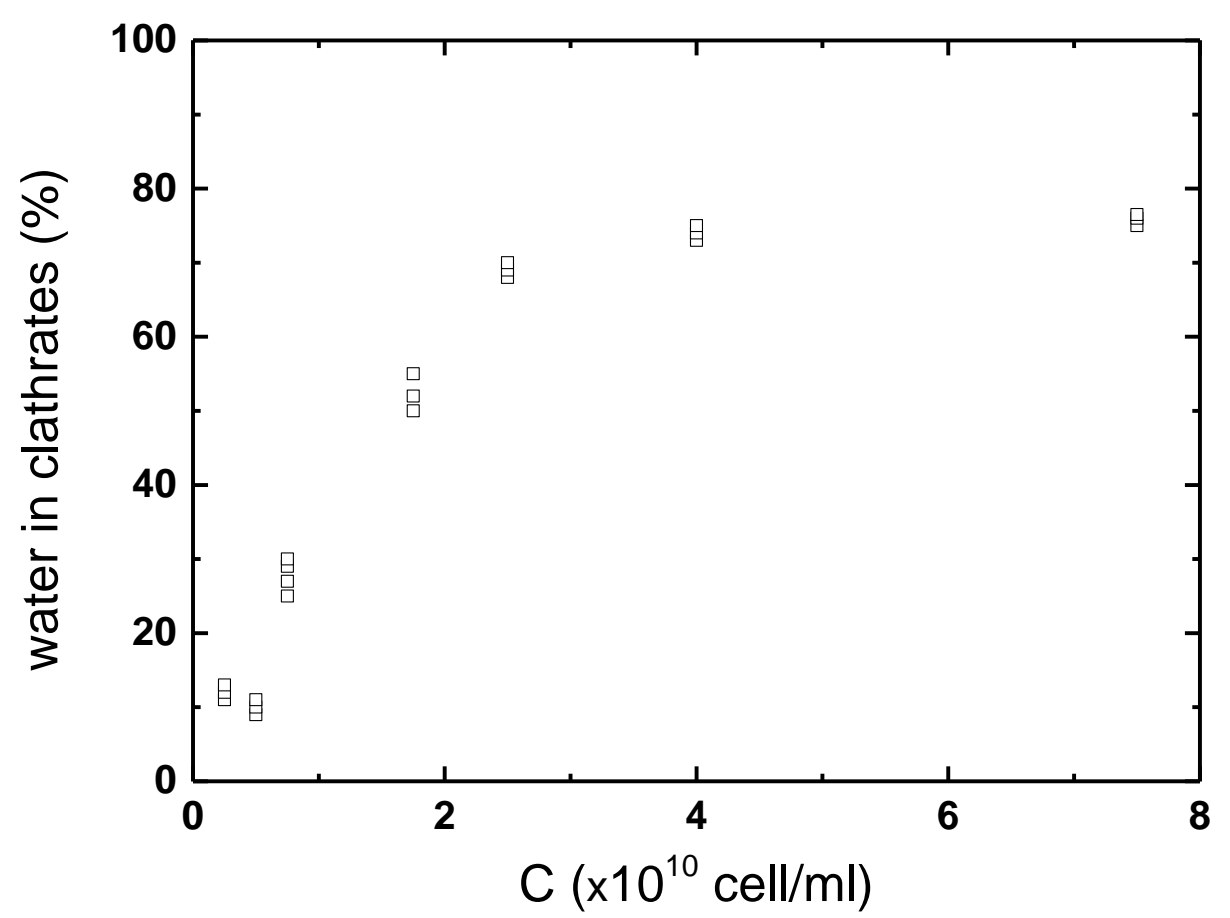

Figure 2. The dependence of the amount of $\mathrm{H}_{2} \mathrm{O}$ molecules bounded within the clathrate structures (i.e., protons of solid phase with $T_{2} \sim 10 \mu \mathrm{s}$ ) on the concentration of cells in the suspensions of E.coli with xenon clathrates. The results were obtained from NMR (FID) measurements taking into account the procedure of Refs. [37, 38]. T = $280 \mathrm{~K}$. Pressure is $1.2 \mathrm{MPa}$. Frequency is $20 \mathrm{MHz}$.

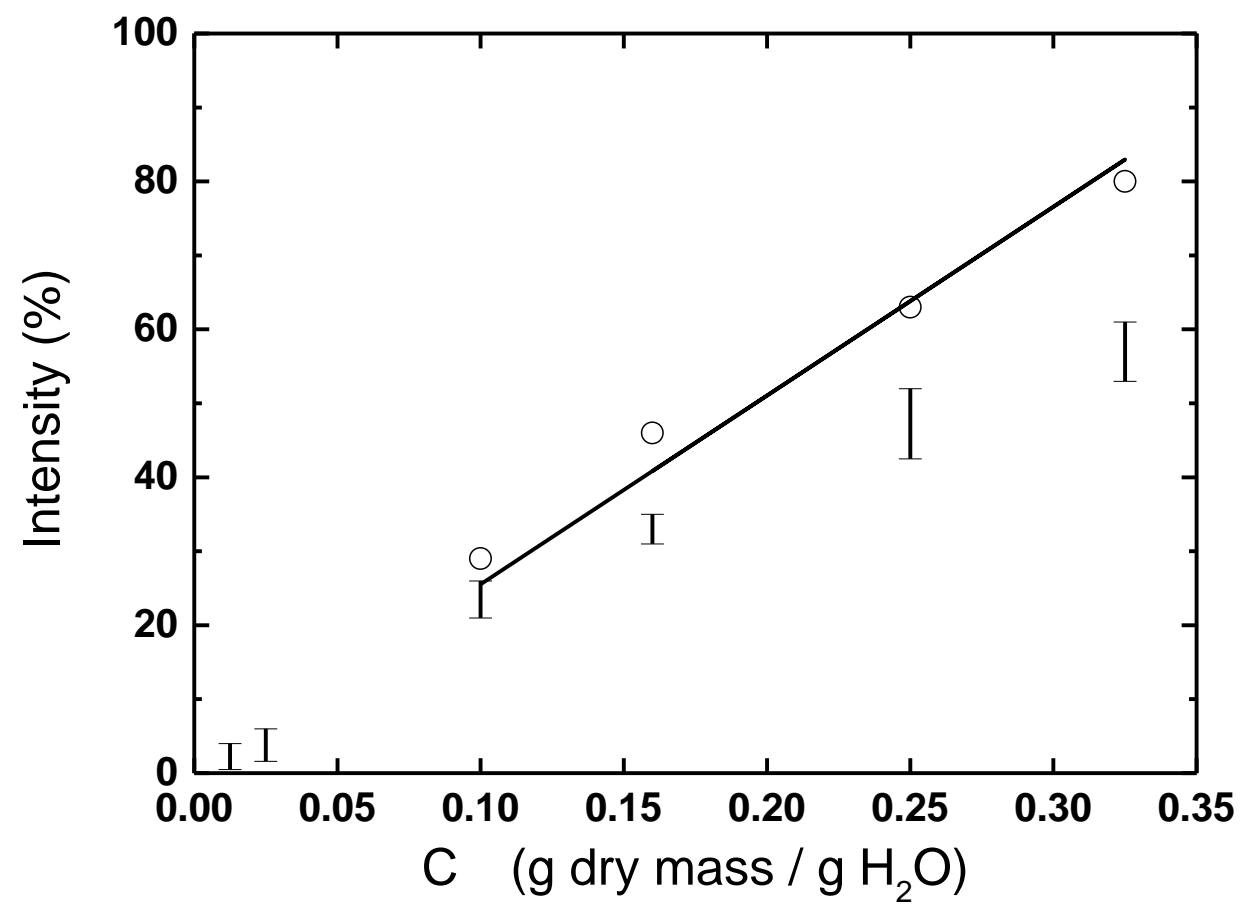

Figure 3. The relative amplitude of fast relaxing $T_{1}$-component (vertical line segments) and calculated fraction of intracellular water (open circles) in the liquid phase of E.coli suspensions with xenon clathrates as a function of cell concentration (C). Solid line is the linear fit to the data (open circles) calculated on the base of summary volume of cells.

$\mathrm{T}=280 \mathrm{~K}$. Pressure is $1.2 \mathrm{MPa}$. Frequency is $90 \mathrm{MHz}$. 
It is worth to emphasize that the dependence of intracellular water amount on the concentration of cells in suspension (Fig.3, open circles) ideally should be a straight line coming through the origin of the coordinates and the meaning which can be obtained at tightly packed cells, i.e., when extracellular water would be completely removed. The experimental curve for the population of the fast relaxing $T_{1}$-component is positioned lower than that based on the total volume of the cells (Fig.3). This could be explained by the osmotic compression of the cell due to an increase in the concentration of solutes in extracellular space upon xenon clathrate formation and a decrease in the amount of remaining liquid water. This is in line with the published data on paramagnetic doping (when the $\mathrm{Mn}^{2+}$ ions are introduced into the extracellular water to separate the proton signals of intracellular and extracellular compartments) in studying the xenon clathrate formation in the extracellular compartments of bacterial suspensions [21, 36].

The behavior of $T_{1}$-components in E.coli suspensions with xenon hydrates depended on the amount of clathrate microcrystals. This suggests that the proton exchange between the extracellular water and the protons of solid clathrate lattice could also affect $T_{1}$-component of the extracellular water. This is in line with some published NMR-data [37, 39, 43, 44, 51]. Ref. [44] showed that the $T_{1}$ of protons of ice was increasing with decreasing the temperature from $273 \mathrm{~K}$ to $213 \mathrm{~K}$. For example, at $T=273 \mathrm{~K}$ the $T_{1}$ of protons of ice was $0.5 \mathrm{~s}$ increasing to $3 \mathrm{~s}$ at $250 \mathrm{~K}$. In contrast to ice the $T_{1}$ of protons of liquid water decreased with decreasing the temperature of water [43, 44, 51]. According to the published data on dispersions of xenon clathrates in pure water $[37,39]$, the temperature dependence of $T_{1}$ for water protons showed a minimum. A proton exchange between liquid water and solid clathrate phase resulted in relaxation behavior of the protons of water molecules displaced among clathrate microcrystals in dispersion. Upon this influence dependence of $T_{1}$ on temperature for the protons of water remaining in liquid state became similar to that for the protons of ice. Thus the protons of liquid water near the crystals of clathrate ice could experience spin-lattice relaxation with increased values of $T_{1}$ exceeded typical values for liquid water. However an interpretation of longitudinal relaxation in E.coli suspension with xenon hydrates could be much harder than it was for clathrate dispersions of xenon in pure water because of additional relaxation sinks and hydration of cell surfaces. For phase of clathrate crystals at the temperatures above $273 \mathrm{~K}$ $T_{1}$ is probably of the order of few seconds (the estimate is based on the mobility of water molecules in ice at $213-273 \mathrm{~K}$ and in clathrate crystals at 275-280 K [25, 26, 30, 38, 44, 54]). Then for the protons of liquid water remaining in extracellular compartment and dispersed between hydrate crystals and bacterial cells we can expect $T_{1}$ to be of the order of $1 \mathrm{~s}$. The amount of water molecules participating in the exchange with clathrate phase was increasing with growth of the amount of hydrate crystals in suspensions as surface of boundaries of water-clathrate ice was growing up. This resulted in changing spin-lattice relaxation time $T_{1}$ for slow component of longitudinal magnetization recovery. In one particular example for E.coli suspension with xenon hydrates, $T_{1}$ doubled compared to the initial spin-lattice relaxation constant of $T_{1}=0.54 \mathrm{~s}$ at a cell concentration of $0.23 \mathrm{~g}$ dry mass $/ \mathrm{g} \mathrm{H}_{2} \mathrm{O}$.

According to the data of Ref. [44], the proton spin-lattice relaxation times in carefully purified ice have been measured for the temperature range of $213-273 \mathrm{~K}$. The authors of this work considered a relationship of $T_{1}$ with rotational jump time $\tau_{\mathrm{j}}$ as follows: $T_{1}=2.14 \times 10^{5} \times \tau_{\mathrm{j}}$. The dependence of $T_{1}$ against inverse temperature has been characterized by single apparent activation energy of $E_{\mathrm{a}} \approx 59 \mathrm{~kJ} / \mathrm{mol}$ [44] (or $E_{\mathrm{a}} \approx 56.3$ $\mathrm{kJ} / \mathrm{mol}$ according to the data published in Ref. [40]) and $T_{1}$ in ice was frequently dependent as $\sim \omega_{0}{ }^{2}$ [44]. So the measurements of $T_{1}$ at different resonance frequencies could also clarify some details in frequently connecting motion of protons in suspensions with xenon hydrates. From the data of Refs. [37, 43] the temperature dependence of $T_{1}$ (an Arrhenius plot $[21,40]$ in the range of 278-286 K) for the protons of water molecules displaced among clathrate microcrystals in dispersion gave the apparent activation energy of $E_{\mathrm{a}}=26.4 \pm 3.7 \mathrm{~kJ} / \mathrm{mol}$. NMR data of Ref. [55] presented apparent activation energy for xenon hydrates as $E_{\text {a }}=24.7 \pm 3.3 \mathrm{~kJ} / \mathrm{mol}$.

In order to estimate correlation times $\tau_{\mathrm{c}}$ (as characteristics of molecular motions based on NMR relaxation time measurements) in E.coli suspensions with xenon clathrates we applied the approach of earlier works [45, 46, 51, 53, 56] assuming the motions involved in intramolecular relaxation and using the expressions reported for $1 / T_{1}$ in terms of the correlation times:

$$
\begin{gathered}
\frac{1}{T_{1}}=\frac{2}{3} C\left\{J\left(\omega_{0}, \tau_{c}\right)+4 J\left(2 \omega_{0}, \tau_{c}\right)\right\} \\
\text { with } J\left(\omega, \tau_{c}\right)=\frac{\tau_{c}}{1+\omega^{2} \tau_{c}^{2}}
\end{gathered}
$$

where $\omega_{0}$ is the Larmor angular frequency in a constant magnetic field $B_{0}$. It is related to the resonance frequency $v_{0}$ by the relation $\omega_{0}=2 \pi v_{0} . C=2.5 \times 10^{10} \mathrm{~s}^{-2}$ is the constant of dipole binding of water molecules.

The use of Eq.(1) can give first estimation of the relaxation behavior of the protons of remaining water molecules displaced among clathrate microcrystals in bacterial suspensions assuming some values of correlation times. According to published data [56-58] $T_{1}$ values for the unfrozen water in some biomaterials (e.g., solutions of agarose or lysozyme) indicate that mobility of water remaining in liquid state can be described by a distribution of correlation times with single peak centered at $\tau_{\mathrm{c}} \sim 10^{-9} \mathrm{~s}$. For the conditions (temperature, pressure) when xenon still did not form clathrates in suspension each water molecule spends part of its time as isotropic water with single correlation time. The correlation between motion of protons from the water molecules and from macromolecules in principle gives rise to the process of cross-relaxation or spin-diffusion. The observed relaxation rates of water protons can be influenced by cross-relaxation rates. Spin-diffusion occurs by way of mutual exchange of 
spin-magnetization between water protons of the hydration water and protons on macromolecules / cell surface. The contribution of cross-relaxation can become very important under the conditions of slow molecular diffusion.

The mobility of water remaining in suspensions after xenon hydrate formation might be comparable with that for unfrozen water in the solutions of biomolecules. This allows a calculation of $T_{1}$ at two experimental resonance frequencies ( 90 and $20 \mathrm{MHz}$ ) at the value of $\tau_{\mathrm{c}}$, for instance, of $2.0 \times 10^{-9} \mathrm{~s}$. The results show reasonable difference $(3-4$ times) in ratio of $T_{1}$-values for 90 and $20 \mathrm{MHz}$ although the value $T_{1} \approx 28 \mathrm{~ms}$ (at $90 \mathrm{MHz}$ ) looks very small in comparison with experimental $T_{1}$-values for extracellular water (0.5-1.2 s) in bacterial suspension with xenon clathrates. For changed value of correlation time (one order) to $\tau_{\mathrm{c}}=2.0 \times 10^{-10} \mathrm{~s}$ the calculation of $T_{1}$ according to Eq.(1) would result in $T_{1}=60-63 \mathrm{~ms}$ (these simulated values are still small in comparison with $T_{1}$ from experiments) showing very small difference between simulated $T_{1}$ values for 90 and $20 \mathrm{MHz}$. The estimation for next change to $\tau_{\mathrm{c}}=2.0 \times 10^{-11} \mathrm{~s}$ was also unsuccessful to match the experimental meanings of $T_{1}$ for two resonance frequencies by the calculated values. This suggests that the motions with correlation times $\tau_{\mathrm{c}} \sim 10^{-9} \mathrm{~s}$ (or less for one-two orders) are not likely to be main input in bacterial suspensions with xenon clathrates. So the approach with one correlation time based on literature data should be definitely changed to distribution of $\tau_{\mathrm{c}}$ to fit the experiments in E.coli suspension with clathrates of xenon.

\subsection{Factors Defining Mobility of Water among Xenon Clathrates in Bacterial Suspension}

The approach of Ref. [56] which studied bound water in frozen erythrocytes by NMR could be probed on bacterial cells adopting the usual formulations of the relationship between relaxation times and correlation times and assuming as well a log-normal distribution of correlation times with distribution function:

$$
P(\tau) d \tau=\frac{1}{\sqrt{\beta \pi}} \exp \left[-\left(\frac{Z}{\beta}\right)^{2}\right] d Z
$$

Here $\mathrm{Z}=\ln \left(\tau / \tau^{*}\right)$ and $\tau^{*}$ is the median of the correlation time distribution and $\beta$ is the distribution width. The authors of Ref. [56] assumed that $\beta$ is independent of temperature and $\tau^{*}$ is dependent of temperature following to Arrhenius expression with activation energy for motion corresponding to the correlation times. The spin-lattice relaxation rate $1 / T_{1}$ is then [56]:

$$
\frac{1}{T_{1}}=\frac{2}{3} C\left[\int_{0}^{\infty} \frac{\tau P(\tau) d \tau}{1+\left(\omega_{0} \tau\right)^{2}}+2 \int_{0}^{\infty} \frac{2 \tau P(\tau) d \tau}{1+\left(2 \omega_{0} \tau\right)^{2}}\right]
$$

For the evaluation of the relaxation rates it is necessary to estimate the integrals in Eq.(3). In Ref. [56] this was done numerically using the relation [57]: $\int_{0}^{\infty} \frac{\omega_{0} \tau P(\tau) d \tau}{1+\left(\omega_{0} \tau\right)^{2}}=\frac{1}{2 \sqrt{\pi}} \int_{-\infty}^{\infty} \exp \left(-\mu^{2}\right)[\cosh (X+\beta \mu)]^{-1} d \mu$

for a log-normal distribution where $X=\ln \left(\omega_{0} \cdot \tau^{*}\right)$.

The approach to fit relaxation data in bacterial suspensions with xenon clathrates is based on certain assumptions (water molecules are undergoing to translational and rotational motions governed by a distribution of correlation times) which allow several adjustable parameters for each correlation time: dipole constant $C, \beta, \tau^{*}$ and activation energy stemming from temperature dependence of $\tau^{*}$. In principle these parameters could be determined by a computer fit of the data using an iterative method. In reality, the dipole constant of water, in other words, the rigid-lattice second moment is in the range from $1.5 \times 10^{10} \mathrm{~s}^{-2}$ for isolated molecule of water to $2.6 \times 10^{10}$ $\mathrm{s}^{-2}$ for crystals of ice [56]. There is indefinite choice for the constant of dipole binding as the relaxation data on $T_{1}$ were obtained for the remaining water in E.coli suspensions after clathrate formation experiencing an exchange with solid phase of clathrate ice.

Fitting the data to the model with one log-normal distribution of correlation times gives the values for the most of the parameters as widely varied. The authors of Ref. [56] for the remaining liquid water in frozen red blood cell (RBC) suspension proposed a model which describes NMR relaxation data by a distribution with two peaks. Additional parameters for the fraction of water molecules under first peak and for the fraction of water molecules under second peak were introduced in order to fit the distribution of $\tau_{\mathrm{c}}$. Analysing the published model for RBC suspensions we suppose that for clathrates of xenon in cell suspensions more consistent model could be developed further. Some additional experiments should involve relaxation of xenon atoms and introduction of parameters responsible for the presence of xenon in suspensions. An idea to explain a cell metabolism during/after xenon clathrate formation could be developed to add the details to total pattern of cell changes. An increase of salt concentration in extracellular water at xenon clathrate formation causes shrinking the cells by loss of free water. When the cells compressed the anion and cation flux across the membrane can be controlled and coupled to the cell metabolism involving a regulation of water structure. The remaining intracellular water can be more highly structured resulting in decreased relaxation times (i.e., increased rates $R_{1}=1 / T_{1}$ ) with cell concentration (Fig.4, Fig.5).

For the future modelling the suspensions with xenon it is necessary also to take into account the changes produced by xenon dissolved in cell suspension. It is possible to consider extracellular water as saline with a concentration $0.9 \%$ by weight. According to the data [59] the solubility of xenon in saline water is low, in particular, Ostwald coefficient is equal to 0.0926 (at standard temperature and $0.101 \mathrm{MPa}$ of gas pressure). However the $T_{1}$ value of xenon is quite long (66 s, when measured at magnetic field $B_{0}=9.4 \mathrm{~T}$ ) due to dipolar couplings with proton spins [59]. 


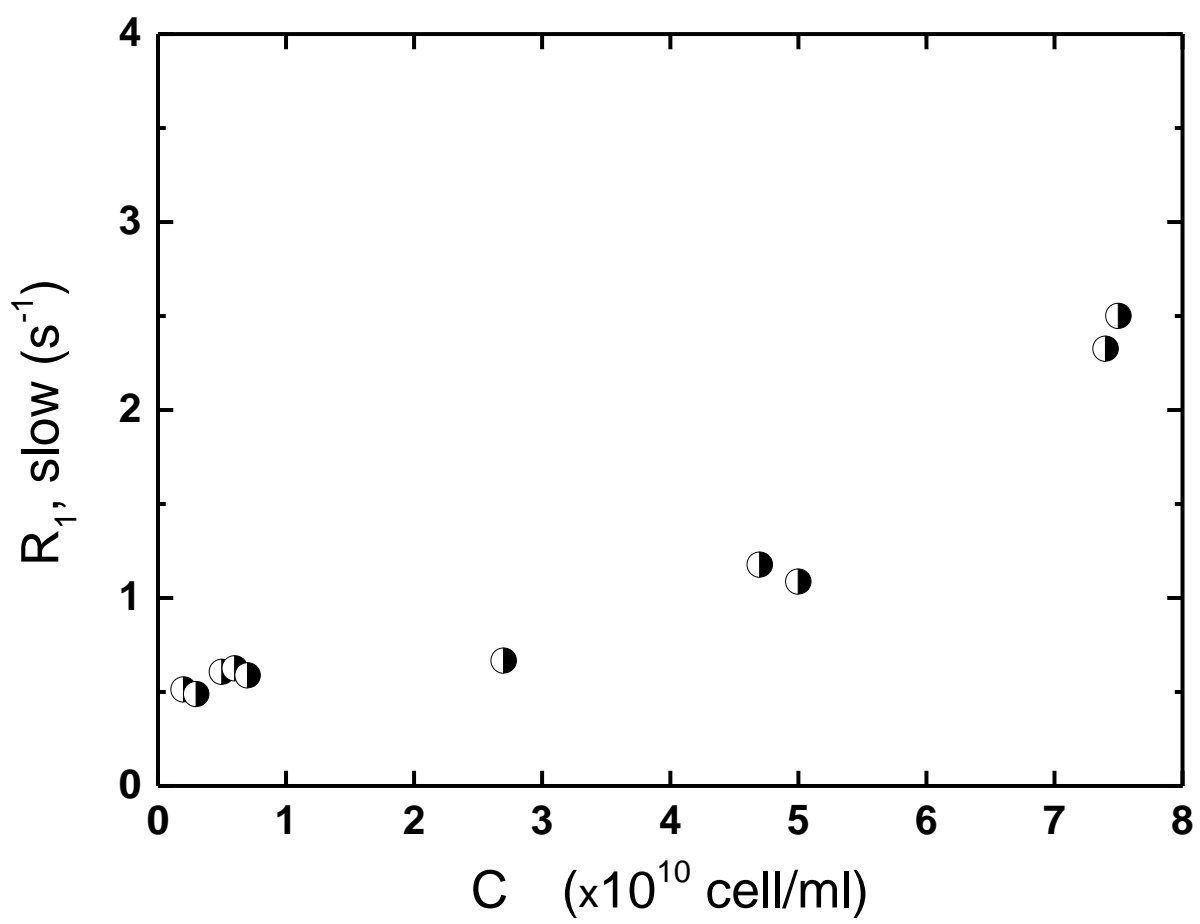

Figure 4. Spin-lattice relaxation rate $R_{1}=1 / T_{1}$ of slow relaxing component of longitudinal magnetization of water protons in liquid phase of E.coli suspensions with xenon clathrates as a function of cell concentration. T = 280 K. Pressure is $1.2 \mathrm{MPa}$. Frequency is $20 \mathrm{MHz}$

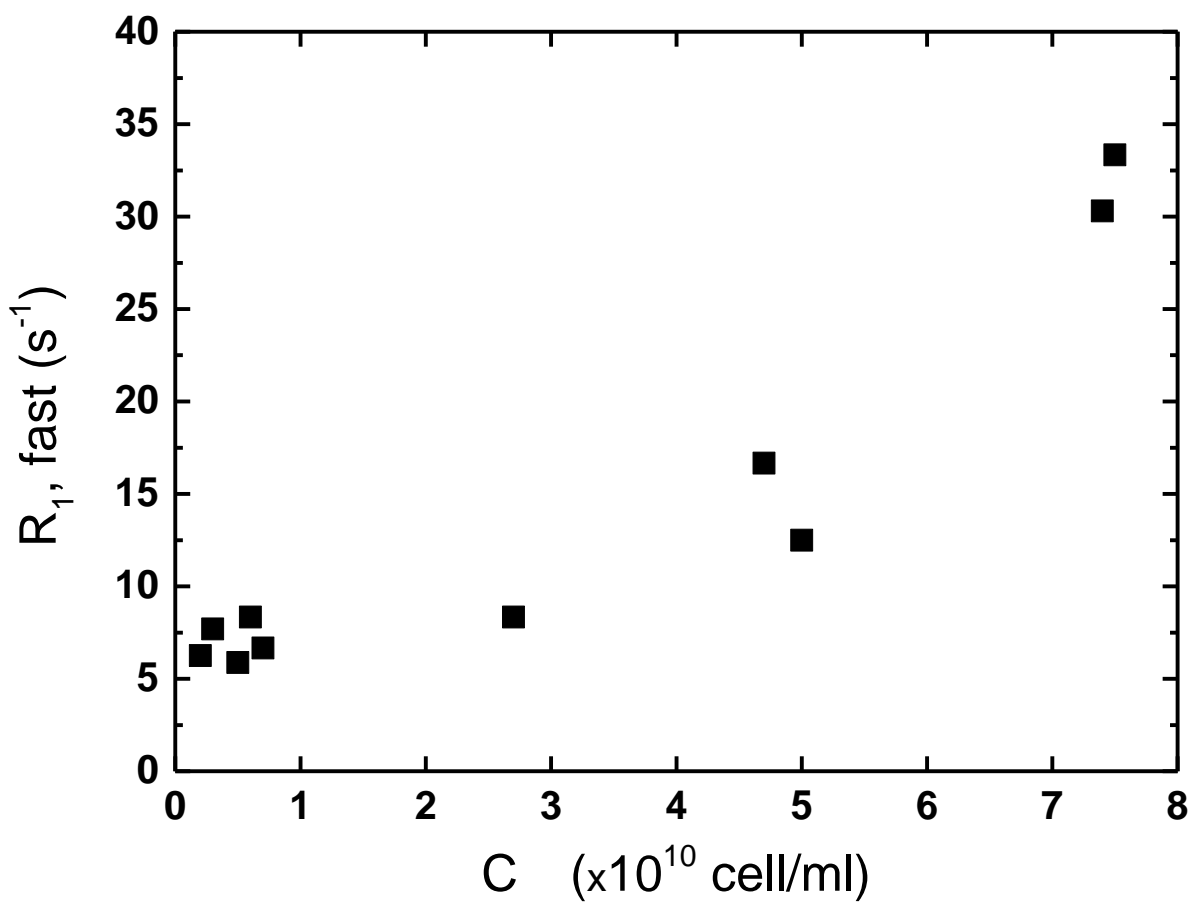

Figure 5. Spin-lattice relaxation rate $R_{1}=1 / T_{1}$ of fast relaxing component of longitudinal magnetization of water protons in liquid phase of E.coli suspensions with xenon clathrates as a function of cell concentration. $\mathrm{T}=280 \mathrm{~K}$. Pressure is $1.2 \mathrm{MPa}$. Frequency is $20 \mathrm{MHz}$ 
An increase of the cell concentration in E.coli suspensions with xenon clathrates resulted in decrease of slow (and fast) components of longitudinal relaxation time $T_{1}$ (Figs.4, 5). This could be associated with increasing viscosity because of the concentration of solutes (inside and outside cells) increased. This is also could be considered as a result of increasing exchange between the protons of liquid water and the protons of macromolecular surface structures of cell in the case of spin-diffusion. Last suggestion is rather unlikely as published data indicated that spin-diffusion affects the relaxation of protons at high concentration of proteins and macromolecules [45, 60-62]. A temperature dependence of slow $T_{1}$ component in the range of 277-289 K (Arrhenius plot) resulted in apparent activation energy $E_{\mathrm{a}}=27.2 \pm 2.0$ $\mathrm{kJ} / \mathrm{mol}$ which exceeds $E_{\mathrm{a}}$-value for pure water $(\sim 21 \mathrm{~kJ} / \mathrm{mol}$ [40]). Probably, a mobility of water molecules in liquid phase of cell suspensions at xenon clathrate formation decreased not only due to increasing cell concentration and compress of cytoplasm but significantly due to dissolution of xenon gas in cells in the amount enough to create separate clathrate cages but not enough to build up a space network of completed clathrate structures. According to the NMR data on ${ }^{129} \mathrm{Xe}$ dissolved in physiological saline at $0.1 \mathrm{MPa}$ and injected into RBC suspension gas-exchange processes between extra- and intracellular compartments occur on the time scale of a few tens milliseconds $[59,63]$. The mixing of xenon between physiological saline and RBCs resulted in accumulation of ${ }^{129} \mathrm{Xe}$ NMR signals inside cells with intensity comparable to that in extracellular compartment for just the period of $\sim 1 \mathrm{~s}$ [59]. However an affinity of xenon to build up the clathrate structures in extra- and intracellular spaces at the temperatures above melting point of ice can be strongly depending on increased gas pressure and other conditions in cells and outside cells including changed metabolism (flux of water and ions via membrane), availability of free liquid water and accumulation of xenon in hydrophobic part of membrane.

\section{Conclusions}

Structural-dynamic changes in bacterial cell properties induced by xenon have been studied by proton spin-lattice relaxation measurements. The inert gas xenon is able to form clathrate hydrates from water molecules at pressure circa 0.4-0.7 MPa and temperature about 276-278 K. An amount of water bound in solid phase of xenon clathrates can be estimated from NMR data. The results clarified how NMR method can monitor a process of clathrate formation and how the xenon atoms and its hydrates interact with cells. The formation of xenon hydrates affected exchange dynamics of water between two compartments and resulted in apparent two $T_{1}$-components. The results of $T_{1}$-measurements showed that the mobility of intra- and intercellular water can be changed at above melting point of common ice with the aid of xenon hydrates. The ability of xenon hydrates to lower water proton relaxation time inside the cells may reflect their propensity to structure and influence on exchange between extracellular and intracellular water.
Detailed interpretation of correlation times as the characteristics of molecular motions in compartments of bacterial suspension in the presence of clathrate ice is not easy because of the lack of suitable mathematical models. However, the results showed that the surface of cells participates in clathrate formation. It affects the dynamics of the water molecules and determines their correlation times.

The changes in mobility of extracellular water at xenon hydrate formation result in changes in cell properties. Molecular mechanisms of these changes (affecting membrane permeability and activity of proteins and lipids) must be further explored involving different NMR facilities. There are many NMR methods for probing the compartments of heterogeneous, in particular, cellular systems. NMR has a useful role in characterizing the molecular properties of separate spaces of cellular suspensions giving a possibility to probe water, solutes and surfaces non-invasively. This allows keeping the biomaterials under xenon conditions further without distortions.

\section{REFERENCES}

[1] Harris, P.D., and Barnes, R. (2008) The uses of helium and xenon in current clinical practice. Anaesthesia, 63(3), 284-293.

[2] Trudell, J.R., Koblin, D.D., and Eger, E.I. (1998) A molecular description of how noble gases and nitrogen bind to a model site of anesthetic action. Anesth. Analg, 87(2), 411-418.

[3] Goto, T., Suwa, K., Uezono, S., et al. (1998) The blood-gas partition coefficient of xenon may be lower than generally accepted. British Journal of Anaesthesia, 80, 255-256.

[4] Steward, A., Allott, P.R., Cowles, A.L., and Mapleson,W.W. (1973) Solubility coefficients for inhaled anaesthetics for water, oil and biological media. British Journal of Anaesthesia, 45, 282-293.

[5] Booker, R.D., Sum, A. K. (2013) Biophysical changes induced by xenon on phospholipid bilayers. Biochimica et Biophysica Acta. 1828, 1347-1356.

[6] Kopp Lugli, A., Yost, C.S., and Kindler, C.H. (2009) Anaesthetic mechanisms: update on the challenge of unravelling the mystery of anaesthesia. Eur.J.Anaesthesiol., 26(10), 807-820.

[7] Yeh, Sh-Y., Peterson, R.E. (1965) Solubility of krypton and xenon in blood, protein solutions, and tissue homogenates. Journal of Applied Physiology, 20(5), 1041-1047.

[8] Sanders, R.D., Ma, D., and Maze, M. (2005) Xenon: elemental anaesthesia in clinical practice. British Med. Bulletin. 71(1), 115-135.

[9] Rodin, V.V., Isangalin, F.Sh., and Volkov, V.Ya. (1982) The state of the water in E.coli cell mixtures in presence of xenon: NMR-relaxation data. In: Proceedings of $1^{\text {st }}$ All-Union Biophysical Congress, M.,vol.2, p.9. 
[10] Rodin, V.V., Isangalin, F.Sh., and Volkov, V.Ya. (1984) The effect of xenon on the state of DNA-bound water systems using a method of NMR. In: Proceedings of $8^{\text {th }}$ Scientific Conference of Research Institute for Applied Microbiology. Obolensk, Moscow region, inv.N 158.

[11] Shin, J., Han, S., and Kang, S-P. (2016) Investigation of xenon and natural gas hydrate as a storage medium to maintain the enzymatic activity of the model proteins. Korean J. Chem. Eng., 33(4), 1345-1351.

[12] Rodin, V.V., Isangalin, F.Sh., and Volkov, V.Ya. (1979) Investigation of the gas hydrates formation in protein solutions using the NMR-pulse method. In: $4^{\text {th }}$ Scientific Conference of Research Institute for Applied Microbiology. Serpukhov, inv.N 12, pp.152-154.

[13] Rodin, V.V., and Volkov, V.Ya. (1981) The xenon clathrates formation in aqueous solutions and cell biological systems. Data of pulsed NMR-method. In: Radiospectroscopy methods in biophysics of cell, edited by Volkov, V.Ya. Obolensk, Moscow region, p.37.

[14] Rodin, V.V., Isangalin, F.Sh., and Volkov, V.Ya. (1984) "Freezing" of the bacterial mixtures at room temperature by xenon clathrates. In: Proceedings of $2^{\text {nd }}$ Symposium "Mechanisms of Cryodestruction and Cryopreservation of Biological Objects". Kharkov, vol.1, p.199.

[15] Rodin, V.V., Isangalin, F.Sh., and Volkov, V.Ya. (1984) Investigation of biopolymer-water system in presence of xenon clathrates using NMR-methods. In: $5^{\text {th }}$ Conference on 'Spectroscopy of Biopolymers'. Book of Abstracts, Kharkov, p.188.

[16] Ando, H., Takeya, S., Kawagoe, Y., Makino, Y., Suzuki, T., and Oshita,S. (2009) In situ observation of xenon hydrate formation in onion tissue by using NMR and powder X-ray diffraction measurement. Cryobiology, 59(3), 405.

[17] Hyman, H.H. (1964) The chemistry of the noble gases. J. Chem. Educ., 41(4), 174.

[18] Potapov, A.V. (2011) The study of equilibrium absorption of xenon and krypton by water emulsion solution, depending on the fat content and temperature of the solvent. PhD Thesis, M., $153 \mathrm{p}$.

[19] Ilyin, I.Y., Tkachman, M.G., et al. (2012) Method of preserving a platelet concentrate under elevated xenon concentration and pressure with refrigeration. US-Patent. US 8158339. Rich Products Corporation. NY.USA.

[20] Ilyin, I.Y., Tkachman, M.G. et al (2014) Storage of platelets under pressure with xenon containing atmosphere. US-Patent. US 8652770 B2, NY.USA.

[21] Rodin, V.V. (1985) Water state and formation of xenon clathrate hydrates in bacterial suspensions as studied by NMR. Research Institute of Biophysics, Pushchino, Moscow region, Russia. PhD Thesis, Candidate of Physics-Maths. Sciences: Biophysics, $167 \mathrm{P}$.

[22] Laptev, D. S., Polezhaeva, T. V., Zaitseva, O. O., Khudyakov, A. N., et al. (2015) Effect of inert gas xenon on the functional state of nucleated cells of peripheral blood during freezing. Human Physiology. 41(2), 206-208.

[23] Ponomarev, A, Makeev, O., Gurevich, L., Rodin, V. (2016) Different xenon conditions for preservation of viable tissue in order to use in regenerative medicine. European Cells and Materials, 31, Suppl. 1, p.382. ISSN 1473-2262.
[24] Ponomarev, A., Makeev, O., Gerasimov, M., Gurevich, L., Rodin, V. (2016) Hypothermic preservation with xenon: impact of the energy pathways. Cryobiology,73, 433-434.

[25] Lafond, P.G., Grim, R.G, and Sum, A.K. (2015) Clathrate hydrate equilibrium modeling: Do self-consistent cell models provide unique equilibrium solutions? Can.J.Chem. 93, 826-830.

[26] Klotz, I.M. (1970) Polyhedral Clathrate Hydrates. In: The Frosen Cell, Eds.C.E.W.Wolstenholme, M.O'Conner. J. and A. Churchil, London, pp.5-21.

[27] Rodin, V.V., Isangalin, F.Sh., and Volkov V.Ya. (1984) Structure of water protein solutions in a presence of xenon clathrates. Cryobiology \& Cryo-Medicine, N14, 3-7.

[28] Pauling, L. (1961). A molecular theory of qeneral anesthesia. Science, 134, N3471, 15-21.

[29] McCabe, M. (1967) Water structure and anaesthesia. The Biochim J., 104 (1), 8-9.

[30] Klotz, I.M. (1970) Water: its fitness as molecular environment. In: Bittar E.F. Membranes and Ion Transport. John Wiley. N.Y., vol.1, pp.93-122.

[31] Klotz, I.M. (1970) Comparison of molecular structure of proteins: helix content, distribution of apolar residues. Arch. Biochim. Biophys, 138(2), 704-706.

[32] Rodin, V.V., Isangalin, F.Sh., and Volkov, V.Ya. (1982) Structure formation in the aqueous protein solutions using xenon clathrates. In: $1^{\text {st }}$ All-Union Biophysical Congress, Moscow, vol.1, p.86.

[33] Rodin, V.V., Isangalin, F.Sh., and Volkov, V.Ya. (1981) Investigation of the gas hydrates in water protein solutions using a method of NMR-relaxation. In: Proceedings "Magnetic resonance in biology and medicine," $2^{\text {nd }}$ All-Union Symposium, Moscow, Russia, pp.115-116.

[34] Rodin, V.V., Isangalin, F.Sh., and Volkov, V.Ya. The simple and mixture gas hydrate formation in solutions as studied by NMR. In: $4^{\text {th }}$ Scientific Conference of Research Institute for Applied Microbiology. Serpukhov, 1979, N 12, pp.150-152.

[35] Rodin, V.V., Isangalin, F.Sh., and Volkov, V.Ya. (1982) The formation of xenon clathrates and its characteristics in bacterial systems by NMR-relaxation. In: $7^{\text {th }}$ Scientific Conference of Research Institute for Applied Microbiology. Obolensk, Russia, N 92, p.39.

[36] Rodin, V.V., Isangalin, F.Sh., and Volkov, V.Ya. (1986) Investigation of formation of the xenon clathrates in E.coli suspensions using the NMR method of paramagnetic doping. Biophysics, 31(2), 274-277.

[37] Rodin, V.V., Izmailova, V. N., and Gaitan, V.I. (1999) Aqueous dispersions of xenon clathrates. Colloid J., 61(6),755-763.

[38] Rodin, V.V., Isangalin, F.Sh., and Volkov, V.Ya. (1984) The xenon clathrate formation as a method of immobilisation of water molecules in cell mixtures. In: Proceedings of $2^{\text {nd }}$ All-Union Conference "Experimental Anabiosis." Riga, pp.51-52.

[39] Rodin, V.V., Chalykh, A.E., and Izmailova, V.N. (1997) Formation of xenon clathrates in water and water solutions: NMR-data. In.: Proceedings "Structure and dynamics of molecular systems", KGU, M., Part.3, pp.3-6. 
[40] Rodin, V.V. (1997) Characterization of the biotechnological dispersion systems using magnetic resonance methods. Moscow State University, M., Russia. PhD Thesis, Doctor of Chem. Sciences: Macromolecular and Colloid Chemistry, $278 \mathrm{p}$.

[41] Taj, M.K., Samreen, Z., Ling, J.X., Taj, I., Hassan, T.M., and Yunlin, W. (2014) Escherichia coli as a model organism. Intern.J.Eng.Res. and Sci\&Technology. 3(2), ISSN 2319-5991.

[42] Feng, P., Weagant, S., and Grant, M. (2002) Enumeration of Escherichia coli and the coliform bacteria. Bacteriological Analytical Manual, $8^{\text {th }}$ Edition, FDA/Center for Food Safety $\&$ Applied Nutrition.

[43] Rodin, V.V., Arkhangel'sky, A.N., Isangalin F.Sh., and Volkov V.Ya. (1984) Formation of xenon clathrates in water and study of their properties by pulsed NMR method. Cryobiology \& Cryo-Medicine. Khar'kov. N14. P. 8-11.

[44] Franks, F., (Ed.) (2012) The physics and physical chemistry of water. Springer Science\&Business Media, 596 p. Nuclear magnetic resonance study on water and ice, p.242.

[45] Rodin, V.V. (2017) Methods of magnetic resonance in studying natural biomaterials. Chapter 53 in: Encyclopedia of Physical Organic Chemistry, First Edition. Edited by Zerong Wang. John Wiley \& Sons, Inc., pp.2861-2908. ISBN 978-1-118-47045-9.

[46] Rodin, V.V. (2004) Magnetic resonance methods. Press MFTI: Moscow, 95 p, ISBN 5-7417-0228-7.

[47] Callaghan, P.T. (2011) Translational dynamics and magnetic resonance. Oxford University Press: Oxford, UK.

[48] Rodin, V.V., and Nikerov, V.A. (2014) NMR relaxation and PFG NMR studies of water dynamics in oriented collagen fibres with different degree of cross-linking, Current Tissue Engineering, 3(1), 47-61.

[49] Fantazzini, P., Maccotta, A., Gombia, M., Garavaglia, C. et al. (2006) Solid-liquid nuclear magnetic resonance relaxation and signal amplitude relationships with ranking of seasoned softwoods and hardwoods. Journal of Applied Physics, 100, 0749071-749077.

[50] Rodin, V.V, McDonald, P.J., and Zamani, S. (2013) A nuclear magnetic resonance pulsed field gradient study of self-diffusion of water in hydrated cement pastes. Diffusion Fundamentals, 18, 1-7.

[51] Belton, P.S., and Ratcliffe, R.G. (1985) NMR and compartmentation in biological tissues. Progress in NMR Spectroscopy, 17, 241-279.

[52] Rodin, V.V. (1989) NMR-method for analysis of the disturbances of bacterial membranes at freezing. In.: Issues of Biotechnology, vol.1, pp.45-49.

[53] Mathur-De Vre, R. (1979) The NMR studies of water in biological systems. Prog.Biophys. molec.Biol. 35, 103-134.

[54] Brox, T., Skidmore, M.L., and Brown, J.R. (2015) Characterizing the internal structure of laboratory ice samples with nuclear magnetic resonance. Journal of Glaciology, 61(225), 55-64.

[55] Bottomley, P.A., Pope, J.M., and Cornell, B.A. (1976) A proton magnetic resonance study of the motion of cage water molecules in the clathrate hydrate of xenon. Mol. Phys., 31(4), 1277-1281.

[56] Zipp, A., Kuntz, I.D., and James, T.L. (1976) An investigation of "bound" water in frozen erythrocytes by proton magnetic resonance spin-lattice, spin-spin, and rotating frame spin-lattice relaxation time measurements. $J$. Magn. Res., 24, 411-424.

[57] Belton, P.S., Packer, K.J., and Sellwood, T.C. (1973) NMR studies of water in striated muscle. II. Spin-lattice relaxation times and the dynamics of non-freezing fraction of water. Biochim.Biophys.Acta, 304(1), 56-64.

[58] Duff, I.D., Derbyshire, W. (1975) NMR of frozen agarose gel. J.Magn. Reson., 17, 89-94.

[59] Bifone, A., Song, Y.-Q., Seydoux, R. et al. (1996) NMR of laser-polarized xenon in human blood. Proc.Natl.Acad.Sci. USA, 93, 12932-12936.

[60] Koenig, S.H., Bryant, R.G, Hallenga, K. et al. (1978) Magnetic cross-relaxation among protons in protein solutions. Biochemistry, 17(20), 4348-4358.

[61] Fung, B. M., and McGaughy, T. W. (1980) Cross-relaxation in hydrated collagen. J. Magn. Reson. 39, 413-420.

[62] Rodin, V. V., Foucat, L., and Renou, J. P. (2004) Natural polymers according to NMR data: cross-relaxation in hydrated collagen macromolecules from two connective tissues, Biofizika, 49(4), 608-616.

[63] Norquay, G., Leung, G., Stewart, N.J. et al. (2016) ${ }^{129} \mathrm{Xe}$ chemical shift in human blood and pulmonary blood oxygenation measurement in humans using hyperpolarized ${ }^{129}$ Xe NMR. Mag.Res.in Med. DOI 10.1002/mrm.26225. 